\title{
Etude par la chaîne de valeur de la filière d'exploitation de Bagrus spp. dans la partie congolaise des Lacs Albert et Edouard
}

\author{
Joseph Matunguru MASIRIKA ${ }^{1,2,3^{*}}$, Gabriel Okito MUKABO ${ }^{2,4}$, Patrick Kiyani \\ KASEREKA $^{3}$, Jonas Uvon JARIEKONG' ${ }^{5}$, Gaston Hamulonge TULINABO ${ }^{6}$, \\ Jean-Claude MICHA ${ }^{7}$, Gaspard NTAKIMAZI ${ }^{2}$ et Venant Muderhwa NSHOMBO ${ }^{8}$
}

\author{
${ }^{1}$ Ecole Doctorale, faculté des Sciences, Université du Burundi, BP 1550 Bujumbura-Burundi. \\ ${ }^{2}$ Centre de Recherche en Sciences Naturelles et de l'Environnement, des Sciences Facultés, Université du \\ Burundi, BP : 2700 Bujumbura-Burundi. \\ ${ }^{3}$ Département de l'Environnement et Développement Durable, Institut Supérieur des Techniques de \\ Développement de Kalehe (ISTD/Kalehe), République Démocratique du Congo. \\ ${ }^{4}$ Section Eaux et Forêts Institut Supérieur d'Agrovétérinaire et de Conservation de la nature de Minembwe, \\ (ISAVC/Minembwe). BP 6295 Bujumbura-Burundi. \\ ${ }^{5}$ Faculté des Sciences Agronomiques Université Shalom de Bunia (USB), BP 304 Bunia, République \\ Démocratique du Congo. \\ ${ }^{6}$ Faculture des Sciences, Université Libre de Bunyakiri (ULBu), République Démocratique du Congo. \\ ${ }^{7}$ Unité de Recherche en Biologie Environnementale et Evolutive, Département de Biologie, Université de \\ Namur. BP 5000 Namur, Belgique et ERAIFT, Kinshasa, RD Congo. \\ ${ }^{8}$ Département de Biologie, Université Officielle de Bukavu (U.O.B./Bukavu). BP 570 Bukavu-RD Congo. \\ *Auteur correspondant ; E-mail : josephmatunguru@gmail.com ; Tel : +243828944000
}

\section{RESUME}

La filière d'exploitation de Bagrus spp. occupe une place importante dans l'économie aux lacs Albert et Edouard. Sa durabilité est compromise par les inégalités dans la distribution des revenus. Cette étude visait à analyser la chaîne des valeurs et d'examiner l'ensemble des coûts financiers le long de cette chaîne afin de déterminer le lieu, les valeurs ajoutées et la contribution relative des différents acteurs à la lumière de l'organisation de la filière et du type de gouvernance qui régit les rapports entre les acteurs. Des enquêtes ont ciblé les acteurs de six chaînes de valeur. Celles-ci ont été choisies sur la base du produit (la nature du marché, de la localisation géographique et du degré d'enclavement). Les principaux résultats sont de trois types de chaînes de valeur : domestique, globale et mixte. Le modèle de gouvernance le plus fréquent s'apparente à un "réseau dirigé". Le pêcheur est le principal générateur de valeur mais ce sont les mareyeurs et commissionnaires qui dominent la filière et captent la plus grande partie des revenus.

(C) 2020 International Formulae Group. All rights reserved.

Mots clés : Chaîne de valeur, Bagrus spp., gouvernance, rentabilité, lac Edouard, lac Albert.

\section{Study by the value chain of the exploitation sector of Bagrus spp. in the Congolese part of Lakes Albert and Edouard}

\section{ABSTRACT}

The Bagrus spp. operating chain plays an important role in the economy of lakes Albert and Edouard. Its sustainability is compromised by inequalities in the distribution of income. This study aimed at analyzing. This study aimed to analyzing the value chain and all the financial costs occurring along this chain in order to 
determine the location, the added values and the relative contribution of the different actors in the light of the organization of the sector and the type of governance that governs relationships between actors. Surveys have targeted actors in six value chains. These were chosen on the basis of the product (the nature of the market, geographic location and degree of isolation). The main results concern three types of value chains: domestic, global and mixed. The most common governance model resembles a "directed network". The fisherman is the main generator of value, but it is the wholesalers and commission agents who dominate the sector and capture most of the income.

(C) 2020 International Formulae Group. All rights reserved.

Keywords: Value chain, Bagrus spp., governance, profitability, lake Edouard, lake Albert

\section{INTRODUCTION}

Le poisson fait partie des ressources les plus importantes dans la vie des populations des pays en développement. Plusieurs études récentes (Luhusu et Micha, 2013 ; Cikwanine, 2014 ; Mushagalusa et al., 2015 ; Mukabo et al., 2017 ; Balole et al., 2018 ; Manala et al., 2020), traitent de la chaine de valeur du poisson sur d'autres lacs de la République Démocratique du Congo mais peu traitent du Lac Edouard.

En République Démocratique du Congo et dans le monde entier, la consommation des poissons et des produits aquatiques est en augmentation constante (FAO, 2020). Qu'ils soient pêchés, élevés ou transformés, la variété de ces produits répond aux attentes d'un nombre toujours croissant des consommateurs séduits par la richesse et la diversité de leurs saveurs tout au long de l'année (Amisi et al., 2020). Pêcheurs mais aussi pisciculteurs, employés des halles à marée, mareyeurs, transformateurs, grossistes, distributeurs et poissonniers développent au fil des saisons tout leur savoir-faire pour offrir aux consommateurs des produits de qualité conformes à leurs attentes (Okito et al, 2017). Le recours à la chaîne de valeur $(\mathrm{CV})$ comme cadre d'analyse des activités de pêche artisanale est un fait relativement nouveau. Le débat actuel est centré sur la question de savoir comment mieux créer et distribuer des gains provenant des activités économiques, et comment le faire dans le contexte des pays en développement (CYE Consult, 2009). La CV décrit la gamme complète des activités qui sont nécessaires pour mener un produit ou un service depuis sa conception jusqu'à sa livraison au consommateur final (Kaplinsky et Morris, 2001). Le concept de CV peut être interprété dans un sens étroit ou large. Au sens étroit, une $\mathrm{CV}$ se réfère à toutes les activités réalisées au sein d'une entreprise (Porter, 1985). L'approche globale, en revanche, s'intéresse à la participation des différents agents (entreprises ou autres) dans une CV externe ou globale (Gereffi et al., 1994).

$\mathrm{La} \mathrm{CV}$ aide à comprendre la distribution des rendements découlant des diverses activités de la chaîne. Or, la distribution des rendements possède à la fois une composante "pouvoir" et une composante "revenu". La première reflète le rapport de forces que les différentes parties utilisent dans la détermination de qui fait quoi dans la chaîne, tandis que la seconde traduit les revenus qui reviennent à chaque partie (Kaplinsky and Morris, 2001). L'examen du secteur de la pêche sous l'angle de la CV révèle des réalités assez récurrentes. Davidsson (2007) montre que la majorité des bénéfices générés tout au long de la $\mathrm{CV}$ mondiale des produits de la mer sont capturés par le commerce de détail, le commerce de gros et le secteur de la transformation secondaire de l'industrie de la pêche. Cette tendance est affichée à la fois dans les pays en voie de développement et dans les pays développés.

En 2006, la FAO a analysé comment les revenus sont répartis le long de la $\mathrm{CV}$ des produits de la mer au niveau de quatre pays 
(Maroc, Islande, Tanzanie et Danemark). Les CVs analysées ont montré que le secteur primaire recevait une part relativement faible de la valeur au détail des produits hautement transformés contre une part plus importante dans les produits moins transformés et les produits frais (Gudmundsson et al., 2006 ; Naji et al., 2015). A partir de 2010, la FAO a procédé à une analyse mondiale des $\mathrm{CV}$ s de la pêche et de l'aquaculture dans 14 pays développés et en développement. Cette étude a mis l'accent sur la petite pêche et a porté principalement sur la transmission des prix dans les CVs. Les facteurs qui déterminent les prix et les marges tout au long de la CV ainsi que la répartition des bénéfices entre les différents agents ont été également analysés. Les principales conclusions montrent que dans toutes les CVs analysées, ce sont les petits pêcheurs et les petits aquaculteurs qui reçoivent les bénéfices économiques les plus faibles relativement aux autres agents de la chaîne. Les transformateurs et les marchés de détail captent plus d'avantages en raison de leur pouvoir de négociation relativement plus élevé (Bjorndal et al., 2014).

La pêche via la commercialisation du poisson peut être plus favorisée avec l'existence des marchés potentiels intérieurs et extérieurs solvables (Nahayo, 2010). Les activités autour du poisson occupent une bonne partie de la population riveraine des lacs Albert et Edouard en République Démocratique du Congo. D'après une étude de SENADEP réalisée en 2018, ce secteur emploie environ 20.140 pêcheurs répartis dans 89 campements de pêcheurs au littoral congolais du lac Albert et 19.314 pêcheurs dans 21 campements ou villages de pêche sur la côte congolaise du lac Edouard. Plus de 80.560 personnes sont impliquées dans les activités connexes telles que la construction des pirogues, le traitement et la commercialisation du poisson au Lac Albert contre 58.023 personnes au Lac
Edouard (Matunguru, 2013; Cikwanine, 2014 ; Balole et al., 2018).

La question était donc de savoir «quel groupe d'acteurs détient un rôle clé dans la filière de Bagrus spp. aux lacs Edouard et Albert ?». L'objectif général de cette étude était d'analyser la chaîne des valeurs et d'examiner l'ensemble des coûts financiers survenant le long de cette chaîne, afin de déterminer où et combien de valeurs sont ajoutées et quelle est la contribution relative des différents acteurs. L'analyse a été conduite à la lumière des modes de gouvernance qui régissent les interactions entre acteurs.

\section{MATERIEL ET METHODES}

Milieu d'étude, période et sites
d'échantillonnage

La présente étude a été effectuée en République Démocratique du Congo en Provinces de l'Ituri et du Nord-Kivu plus précisément aux lacs Albert (entre 1'39'33', Nord et 30'51'33' Est) et Edouard (entre $0^{\circ} 20^{\prime}$ Sud et $29^{\circ} 36^{\prime}$ Est). La récolte de données pour chacun de ces deux lacs a été effectuée pendant trente (30) jours ouvrables, soit d'Août à Septembre 2019. Trois sites d'échantillonnage ont été choisis pour chacun de ces deux lacs faisant au total 6 sites. Pour le lac Edouard il s'agit des: Vitshumbi au sud situé dans le territoire de Rutshuru, Kisaka à l'ouest en territoire de Lubero et Kyavinyonge à l'extrême Nord du lac en territoire de Beni (Figure 1). Les sites retenus au lac Albert sont : Mahagi Port situé en territoire de Mahagi, Tchomia en territoire de Djugu et Kasenyi en territoire d'Irumu (Figure 2).

\section{Analyse de la chaîne de valeur (CV)}

Aux lacs Albert et Edouard (LAE), le Bagrus spp est principalement capturé à travers deux types de pêche : la pêche à la palangre et la pêche au filet maillant. Pour la pêche au filet maillant, chaque pirogue (Unité d'Effort de Pêche) compte 7 membres d'équipage : un 
armateur et 6 pêcheurs qui travaillent alternativement en deux équipes de trois. Ces unités d'effort de pêche capturent principalement deux espèces de Bagrus: Bagrus docmak (Forsskall, 1775) et B. bajad (Forsskall, 1775) dont B. docmak est la plus abondante au lac Edouard alors que B. bajad domine au lac Albert. Les données récoltées concernent les deux espèces mais sans les distinguer.

La filière de pêche artisanale englobe un grand nombre de CVs. La filière est considérée ici comme étant constituée "d'une suite d'opérations (ou de transformations), d'une suite d'acteurs et d'une suite de marchés, ce qui implique des flux physiques et leur contrepartie monétaire... ainsi que des comportements d'acteurs guidés par leur intérêt économique " (Bockel and Tallec, 2005a). L'approche de la CV s'intéresse, davantage, à identifier l'ensemble des coûts financiers et économiques le long de la chaîne, afin de déterminer où et combien de valeur est ajoutée et quelle est l'importance relative des différents acteurs, en relation avec la structure de gouvernance formelle et informelle (Gereffi et al., 1994). Les agents de la filière (appelés acteurs) concourent à la production et/ou à la transformation des produits. Selon les cas, il peut s'agir d'une personne physique (pêcheur, commerçant, etc.) ou d'une personne morale (unité de pêche, entreprise de mareyage, usine de transformation, etc.). Par extension, on utilisera aussi le terme « acteur » pour parler de l'ensemble des agents d'un même type.

Etant donné que les chaînes de valeurs impliquent la répétitivité des interactions entre acteurs, la gouvernance pourrait être le reflet de l'organisation des activités dans la chaîne (Kaplinsky and Morris, 2001). En outre, les rapports entre acteurs peuvent aller des relations très dépendantes, où une seule partie domine, à des relations équilibrées, où toutes les parties concernées ont un certain pouvoir qu'ils peuvent exercer. Gereffi (1994) a fait la distinction entre deux types de CVs: les chaînes tirées par les acheteurs et les chaînes contrôlées par les producteurs. L'analyse de l'efficacité de la $\mathrm{CV}$ exploite deux notions essentielles : la valeur ajoutée (VA) créée et les revenus distribués. Ainsi, l'analyse de l'efficacité vise à : i) apprécier le montant de la VA pour l'ensemble de la chaîne ; ii) déterminer comment la VA est créée par la chaîne et par quels acteurs ; et iii) déterminer les revenus distribués, c'est-à-dire, savoir comment sont rémunérés les acteurs pour leur participation aux activités de la chaîne (Bockel and Tallec, 2005b).

Pour recueillir les informations qui servent à l'analyse financière et à l'efficacité d'une CV, des comptes de productionexploitation ont été élaborés pour chaque type d'acteur. Par la suite, et afin de disposer d'une information globale sur l'ensemble de la filière de pêche artisanale dans la zone d'étude, il a été procédé à l'établissement des comptes consolidés pour, d'une part, chacune des sousfilières (nature de Bagrus spp. vendue) identifiées, et ensuite, pour l'ensemble de la filière de pêche Bagrus spp. aux lacs Albert Edouard (LAE). La richesse créée sous forme de VA constitue une source de revenu qui sera distribuée aux quatre acteurs économiques fondamentaux impliqués dans la filière de pêche artisanale. Les ménages reçoivent une partie de la VA sous forme de rémunération du facteur travail (frais de personnel). Les institutions financières perçoivent des frais financiers. Les administrations lèvent des taxes et des impôts. Enfin, les entreprises non financières captent ce qui reste de la VA sous forme de résultat net, après déduction des amortissements (Bockel and Tallec, 2005b). Dans cet article, la notion de revenu couvre toutes les catégories de rémunération. Mais, l'accent est mis davantage sur le revenu des travailleurs individuels (Coût du salaire) et sur le revenu des acteurs en tant que structures de 
gestion des unités de production (Profit Net Estimé).

\section{Enquête auprès des acteurs de la filière}

L'enquête a démarré par la sélection des CVs les plus pertinentes et s'est poursuivie par la typologie des acteurs, l'échantillonnage, la conception des outils de collecte des données, les enquêtes de terrain et le traitement des données recueillies.

\section{Sélection des chaînes de valeur.}

Le choix des CVs a tenu compte de trois critères de stratification : le type de produit (Bagrus frais, salé et fumé), le type de marché (rural ou urbain), et le degré d'enclavement des sites de pêche à partir desquels partent les produits: Faible (port de pêche) ; Moyen (village de pêche situé à proximité des voies routières) ; Elevé (sites de pêche éloignés et dont l'accès est difficile). En s'appuyant sur une bonne connaissance du terrain, ces critères de stratification ont été croisés et les combinaisons redondantes ou inutiles ont été éliminées pour finalement déboucher sur la sélection empirique d'un groupe de six strates ou circuit de CV.

\section{Taille de l'échantillon}

Dans cette étude, nous avons recouru à deux méthodes. La méthode empirique appuyée par les recherches documentaires relatives au thème de recherche et la méthode accélérée de recherche participative. Cette méthode a été utilisée pour récolter les données informatives en rapport avec la filière d'exploitation de Bagrus spp. aux lacs Albert et Edouard à la fois par observation participative et par entretiens semi-structurés. Nous avons aussi recouru à la méthode quantitative soutenue par la technique d'enquête par sondage à choix raisonné (Grawitz in Mukabo et al., 2017), car celle-ci permet de mener une étude sur une partie de la population qui a les mêmes caractéristiques ou qui exerce les mêmes activités. Les questionnaires ont été présentés en module selon la catégorie de chaque acteur intervenant dans la filière d'exploitation de Bagrus spp. aux lacs Albert et Edouard. Pour les pêcheurs, un échantillon de taille minimale de $10 \%$ a été prélevé de façon aléatoire et simple au sein de chaque CV. Sur une population de 568 personnes actives au lac Albert, 191 individus ont aléatoirement été sélectionnés pour autant qu'ils participent dans la CV de Bagrus spp. aux LAE. 249 personnes sur un total de 666 inventoriées au lac Eduard ont été choisies pour répondre à nos questionnaires. Les autres types d'acteurs (mareyeurs, commissionnaires, unité de valorisation, grossistes et détaillants) ont été examinés à l'exhaustivité. Au sein de chaque groupe d'acteurs, la méthode de recueil des données la plus appropriée a été adoptée : enquête par questionnaire, groupe de discussion et interviews. Le nombre d'unités débarquant, les Captures (kg) par Unité d'Effort de Pêche (CPUE : 1 nuit de pêche par catamaran) et le prix de vente des poissons sur la plage sont enregistrés. Les statistiques quotidiennes de Captures par Unité d'Effort de pêches (CPUE) nous sont fournies par un enquêteur affecté à chaque site par les inspections des Pêches. Sur le terrain, le poids du poisson était mesuré avec une balance, précision à $1 \mathrm{~g}$ près.

\section{Méthodologie d'analyse des résultats}

Après le dépouillement, les données collectées auprès de différents acteurs de la filière poisson ont été saisies avec Microsoft Excell 2017. L'analyse s'est faite, après encodage, à l'aide du programme Statistical Package for Social Science (SPSS) for Windows version 12.0. La cartographie a été faite à l'aide du logiciel ARCGIS. Pour les analyses statistiques, le logiciel SPSS 14.0 sous Microsoft Windows 10 a été utilisé. L'ANOVA 1 (One-Way) a été utilisée pour la comparaison des différentes moyennes 
observées sur l'ensemble des variables. L'analyse comptable concerne les comptes d'exploitation des pêcheurs, des mareyeurs, des grossistes, du transporteur et de celui des détaillants au marché. Pour cela, nous avons considéré un même prix moyen d'un Bagrus spp. frais de poids moyen $(0,62 \mathrm{~kg})$ sur l'ensemble de deux lacs pour chacun des acteurs du circuit de commercialisation. Ce prix est de $1900 \mathrm{FC}$ (francs congolais) soit 1,2 \$US pour le pêcheur, il est de 2500 FC (soit 1,6 \$US) pour les Mareyeurs, 2800 FC soit 1,8 \$US pour les commissionnaires, $3300 \mathrm{FC}$ (soit 2,1 \$US) pour le grossiste, et $3950 \mathrm{FC}$ (soit 2,5 \$US) pour le détaillant.

Pour arriver à déduire la marge bénéficiaire d'une unité artisanale de pêche dans le site étudié et en sortir le revenu net par artisan pêcheur, quelques indicateurs économiques ont été calculés à partir d'une unité type (Njifonjou, 2010 in Mukabo et al., 2017). Ces indicateurs sont: Capital Investi (CI): Somme d'achat de tous les matériels constituant l'unité de pêche, Coût Opérationnel (CO): Ensemble de toutes les dépenses, Coût d'Exploitation (CE) : CO Amortissement, Profit brut estimé (PBE) : total des revenus obtenus après-vente, Profit net estimé (PNE) : Marge bénéficiaire de l'unité de pêche (PBE - CE), Taux de rentabilité (TR): Indice permettant de calculer le délai de récupération du capital investi (PNE/C.I *100) et Coût salarial $(C S)=$ revenu par pêcheur. Il n'a pas été tenu compte des dépenses familiales, de celles hors activités, des pertes de captures et de l'autoconsommation.

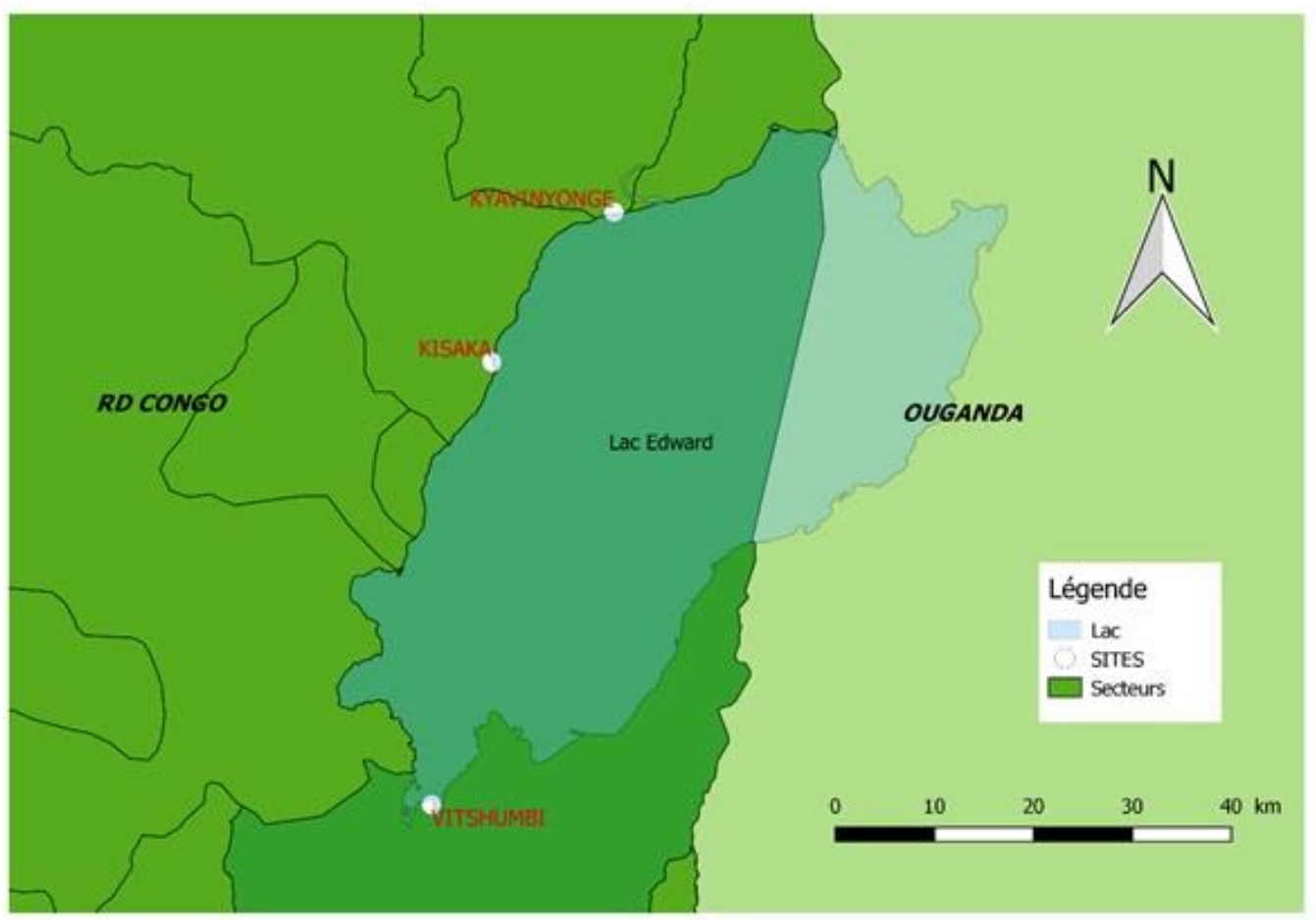

Figure 1 : Sites d'échantillonnage du lac Edouard. 


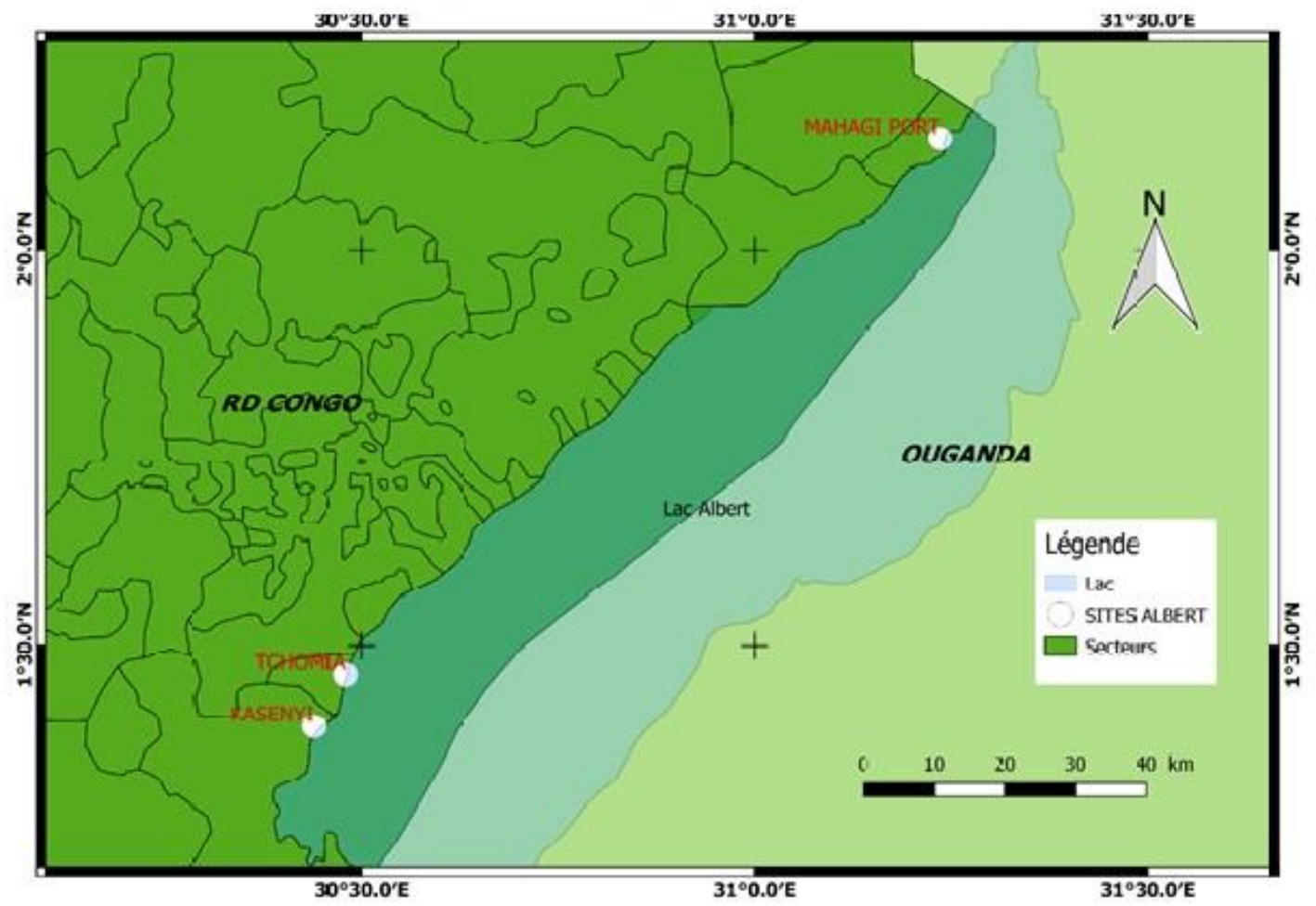

Figure 2 : Sites d'échantillonnage du lac Albert.

\section{RESULTATS}

Caractérisations sociales des différents acteurs intervenant dans la filière de Bagrus spp.

Les résultats (Tableau 1) montrent qu'un total de 1.497 personnes ont participé à cette étude dont 699 acteurs au lac Albert et 798 au lac Edouard. Ces acteurs sont représentés par les pêcheurs, les mareyeurs, les vendeurs (grossistes et détaillants), transporteurs, consommateurs et agent du département de la pêche affectés à chaque site.

\section{Circuit de commercialisation de Bagrus spp.}

En ce qui concerne la nature des Bagrus vendus dans la partie congolaise des lacs Albert et Edouard, les résultats (Figure 3) montrent que : les pêcheurs tant du lac Albert que du lac Edouard préfèrent la vente des Bagrus spp. frais avec un taux respectif de $67 \%$ et $93 \%$. La vente des Bagrus salés et fumés vient en deuxième et troisième position au lac Albert avec un taux respectif de 19 et $12 \%$, tandis qu'au lac Edouard, c'est l'inverse ; les Bagrus fumés $(5 \%)$ occupent la deuxième place dans la vente suivis de Bagrus salés (2\%).

Interrogés à ce sujet, les pêcheurs justifient leur premier choix par le fait que l'état salé et l'état fumé exigent des coûts supplémentaires estimés en termes temporel et économique, parfois difficiles à récupérer étant donné qu'il n'y a pas assez de différences entre le prix des poissons frais et celui des poissons salés ou fumés qui ont perdu de l'eau et donc du poids, sur le marché local. En plus, Bagrus spp. est vendu à la pièce.

\section{Organisation de la Chaîne de valeur pour la filière Bagrus spp. aux LAE}

D'après la Figure 4, six circuits commerciaux appartenant à trois chaînes de valeurs ont été observés le long de la filière de Bagrus spp. aux lacs Edouard et Albert. Les chaînes de valeurs domestiques (Circuits 1 et 
2), les chaînes de valeurs tournées vers les marchés urbains (Circuits 3 et 4), et les chaines de valeurs à caractère mixte dont la production sera destinée tantôt aux marchés urbains, tantôt aux marchés domestiques (Circuits 5 et 6 ). La longueur du circuit est non seulement liée à l'augmentation des opérateurs intervenant dans la chaîne mais aussi aux sources d'approvisionnement et à la nature du poisson vendu.

Pour un même site de pêche, l'étendue et la complexité des chaînes de valeurs dépendent de la destination finale du produit, selon qu'il soit distribué au niveau du marché urbain ou bien local. Au moment où le segment local de la chaîne de valeur destinée aux marchés locaux présente une étendue relativement courte, la chaîne de valeur du poisson destiné au marché urbain arbore des circuits d'une diversité qui n'a d'égal que la multiplicité des agents qui y sont impliqués.

\section{Rôles et relations des acteurs de la filière Bagrus spp. dans la chaîne des valeurs \\ Les enquêtes ont révélé que} l'approvisionnement régulier des pêcheurs en intrants est tributaire de la nature des relations qui lient le pêcheur au mareyeur. En effet, les pêcheurs sont très dépendants de «leur » mareyeur. Par conséquent, ils optent pour une stratégie de fidélisation traduite par un arrangement informel. Cette relation peut aller jusqu'à l'octroi d'une procuration, signée par le pêcheur, qui permet au mareyeur de vendre le poisson à la criée et de bénéficier de la documentation commerciale exigée par les autorités douanières. Tous ces facteurs accentuent la dépendance des pêcheurs vis-àvis des mareyeurs et réduisent leur champ d'autonomie.

Les relations qu'entretiennent les différents acteurs de la filière de pêche aux LEA sont assez complexes. Plusieurs situations sont rencontrées dans cette relation: i) $L a$ confiance (parenté, amitié et voisinage) qui marque, par exemple, les relations tissées par les intermédiaires avec les marins pêcheurs; ii) La concurrence qui s'exprime au niveau des pêcheurs par la recherche du maximum de revenus et au niveau des mareyeurs par le maintien, la consolidation et l'extension de leur propre portefeuille de fournisseurs; iii) $L a$ méfiance à l'égard des intermédiaires: les pêcheurs estiment que les mareyeurs les exploitent en leur proposant des prix faibles alors qu'ils sont détenteurs de la production et ne peuvent pas directement accéder au marché. Le préfinancement au quotidien des opérations de pêche vient asseoir cette prééminence des mareyeurs ; iv) La domination et la tutelle d'un petit nombre de mareyeurs sur les circuits de commercialisation des produits de la pêche. La domination évoquée par les mareyeurs concernant leur relation avec les unités de valorisation (transformation): les mareyeurs déplorent le pouvoir des transformateurs et des conditionneurs qui fixent unilatéralement le prix auquel ils achètent le poisson.

\section{Création et distribution de la valeur au sein de la filière Bagrus spp.}

Les résultats du Tableau 2 montrent qu'une unité de pêche artisanale au lac Albert comme au lac Edouard nécessite un investissement d'au moins 1.345 \$US. Ses coûts d'exploitation s'élèvent à \pm 7.016 \$US. Ce Tableau 3 indique que la capture par unité d'effort (moyenne annuelle) de Bagrus spp. aux lacs Albert et Edouard du côté congolais est de $21 \mathrm{~kg} / \mathrm{nuit}$ au Lac Albert contre 23 $\mathrm{kg} / \mathrm{nuit}$ au lac Edouard.

Après la vente de ces produits de pêche, le profit net estimé (P.N.E.) par unité de pêche artisanale s'élève à $1.552 \pm 451$ \$US avec un Coût Salarial (C.S) d'environ 260 \$US par pêcheur et par mois Au lac Edouard, le Profit Net estimé (P.N.E.) est de $2368 \pm 530$ \$US avec un coût salarial (C.S.) par pêcheur et par mois de $395 \$$.

L'examen de la Figure 5 révèle que les acteurs qui créent le plus de valeur ne sont pas forcément ceux qui en captent la part la plus 
importante sous forme de rémunération. Ainsi, les unités de pêche artisanale viennent en tête de par leur contribution dans la valeur ajoutée totale avec $32 \%$; elles ne captent en retour que $12 \%$ de la masse totale des revenus distribués. Les mareyeurs et commissionnaires arrivent en deuxième et troisième position avec respectivement une contribution de $23 \%$ et $20 \%$ dans la valeur ajoutée totale, mais ils perçoivent en contrepartie $13 \%$ et $17 \%$ des revenus distribués. En quatrième position viennent les grossistes avec une contribution de $15 \%$ de valeur ajoutée et $25 \%$ de masse totale des revenus distribués. Enfin, les détaillants reçoivent une part importante 33\% des revenus alors qu'ils n'ont apporté que $10 \%$ de la valeur ajoutée.

Il ressort de la Figure 6, qu'en moyenne, les unités de valorisation sont les mieux rémunérées. La valeur moyenne du résultat net d'une unité de valorisation est de 4270 \$ USD. Il s'agit des bénéfices réalisés par l'acteur gestionnaire de l'unité de traitement des produits issus de la pêche artisanale. A l'autre extrémité de la filière, les unités de pêche perçoivent les revenus annuels les plus faibles.

\section{Contraintes et Opportunités de la filière d'exploitation de Bagrus spp.}

Le Tableau 4 présente les contraintes et opportunités majeures des maillons de la chaine de valeur ajoutée. L'inexistence de contrat de service, l'insuffisance des connaissances techniques, le faible niveau d'organisation des acteurs et le manque d'appui financier sont les contraintes rencontrées dans les différents maillons de la chaine de valeur. L'absence de crédit bancaire et le manque d'investissement constituent les contraintes majeures identifiées. Les principales opportunités proviennent de l'existence du marché d'écoulement du poisson frais du fait de la forte demande intérieure, de la mise en place d'organisation professionnelle agricole, d'un cadre institutionnel pour le renforcement des capacités des acteurs grâce, aux projets de développement en aquaculture et des structures d'encadrement et de recherche.

Tableau 1 : Différents acteurs de la filière Bagrus spp. aux lacs Albert et Edouard.

\begin{tabular}{lcccccccc}
\hline Lac & \multicolumn{3}{c}{ Albert } & \multicolumn{5}{c}{ Edouard } \\
\hline Sites & Tchomia & Kasenyi & $\begin{array}{c}\text { Mahag } \\
\text { i port }\end{array}$ & S-tot & Kyavinyonge & Kisaka & Vitshumbi & $\begin{array}{c}\text { S- } \\
\text { tot }\end{array}$ \\
& & & & & & & & \\
Vendeurs Equip. & 3 & 5 & 5 & $\mathbf{1 3}$ & 5 & 5 & 5 & $\mathbf{1 5}$ \\
Pêcheurs & 61 & 50 & 80 & $\mathbf{1 9 1}$ & 100 & 50 & 99 & $\mathbf{2 4 9}$ \\
Mareyeurs & 50 & 52 & 50 & $\mathbf{1 5 2}$ & 50 & 50 & 50 & $\mathbf{1 5 0}$ \\
Commissionnair & 40 & 40 & 40 & $\mathbf{1 2 0}$ & 40 & 40 & 40 & $\mathbf{1 2 0}$ \\
es & 27 & 30 & 30 & $\mathbf{8 7}$ & 27 & 28 & 30 & $\mathbf{8 6}$ \\
Grossistes & 52 & 60 & 21 & $\mathbf{1 3 3}$ & 73 & 50 & 52 & $\mathbf{1 7 5}$ \\
Détaillants & 1 & 1 & 1 & $\mathbf{3}$ & 1 & 1 & 1 & $\mathbf{3}$ \\
Autorités & $\mathbf{2 3 4}$ & $\mathbf{2 3 8}$ & $\mathbf{2 2 7}$ & $\mathbf{6 9 9}$ & $\mathbf{2 9 7}$ & $\mathbf{2 2 4}$ & $\mathbf{2 7 7}$ & $\mathbf{7 9 8}$ \\
\hline Totaux & & & & & &
\end{tabular}




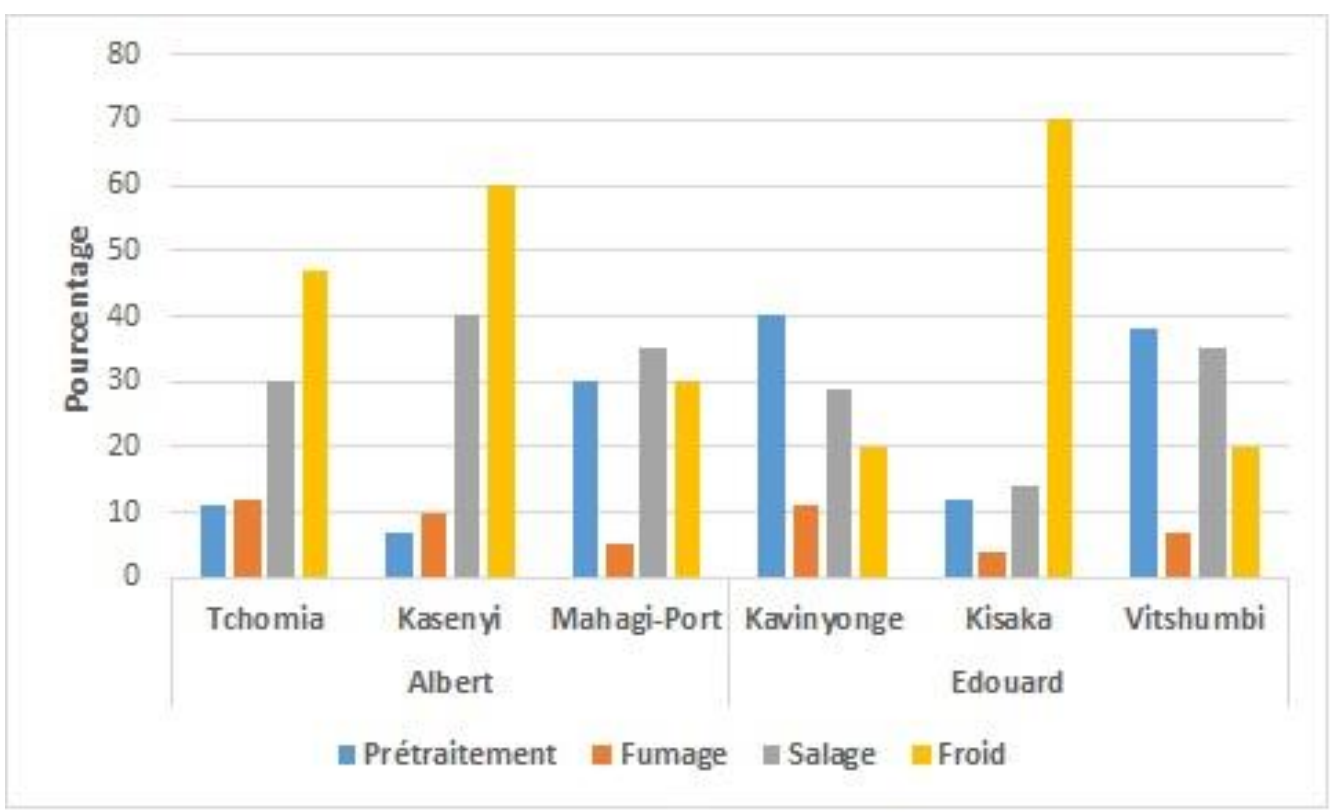

Figure 3 : Méthodes de traitement et conservation de Bagrus spp. aux lacs Edouard et Albert.

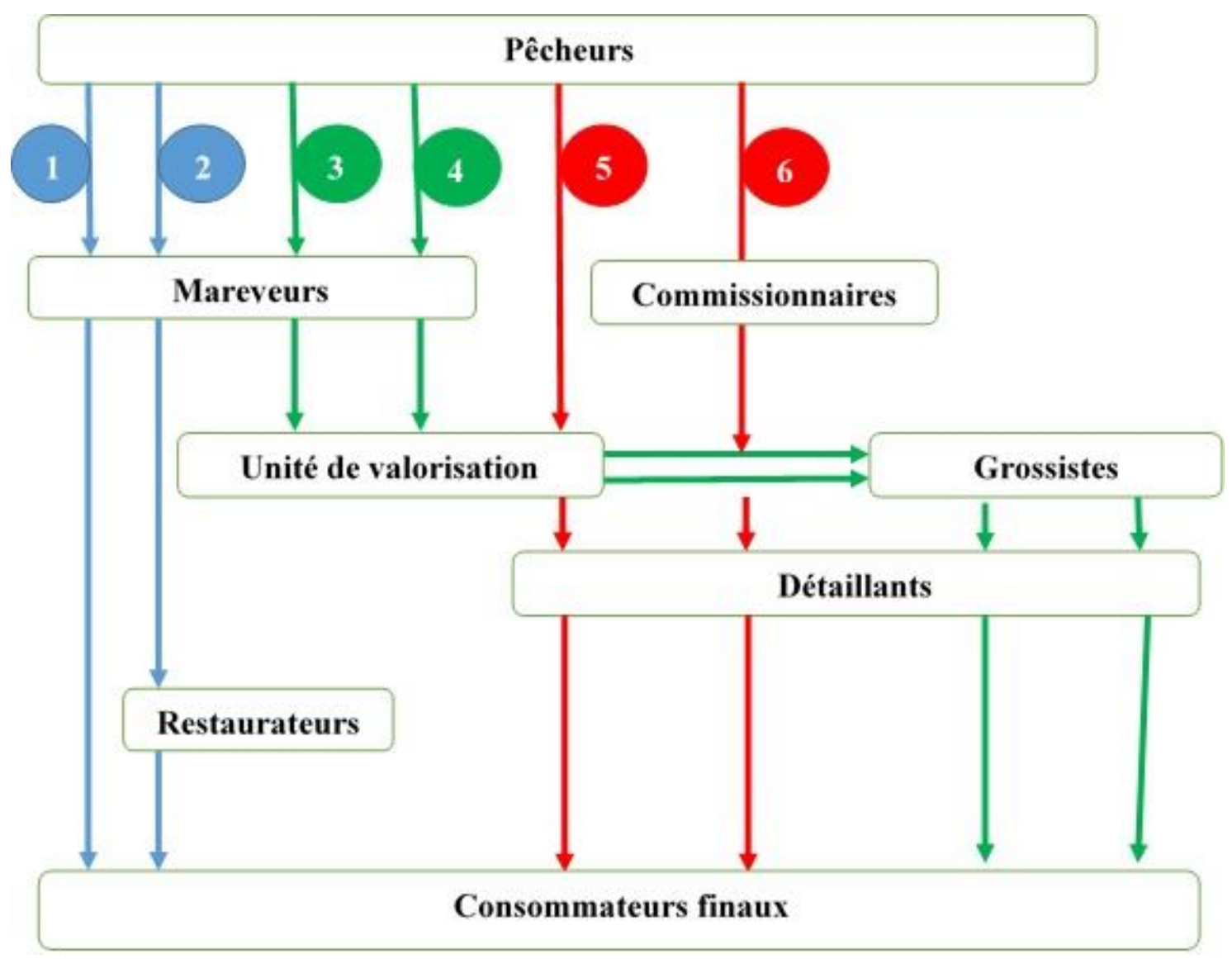

Figure 4 : Circuits (1 à 6) de commercialisation de Bagrus spp. aux lacs Edouard et Albert. 
Tableau 2 : Capture par Unité d’Effort de Pêche (CPUE) et Rentabilité Economique (en \$US).

\begin{tabular}{l|ccccccc}
\hline Lac & CPUE en Kg & P(N/m) & P.B.E & P.N.E. & N.P. & C.S. & T.R \\
\hline Albert & $21 \pm 7$ & $20 \pm 3$ & $8568 \pm 1030$ & $1552 \pm 451$ & $3 \pm 1$ & $260 \pm 140$ & $115 \%$ \\
& & & & & & & \\
\hline Edouard & $23 \pm 8$ & $20 \pm 2$ & $9384 \pm 1400$ & $2368 \pm 530$ & $3 \pm 1$ & $395 \pm 150$ & $176 \%$ \\
\hline
\end{tabular}

La Capture par Unité d'Effort de Pêche (CPUE) et rentabilité économique (en \$US) de l'unité de pêche artisanale des Lacs Albert et Edouard pour Bagrus spp (moyenne \pm écart-type) (UPA = Unité de pêche artisanale, $\mathrm{P}(\mathrm{N} / \mathrm{m})=$ Pêche (Nuits/mois), P.B.E. $=$ Profit Brut Estimé, P.N.E. $=$ Profit Net Estimé, N.P= Nombre de pêcheurs, C.S.$=$ Coût Salarial, T.R. $=$ Taux de Rentabilité).

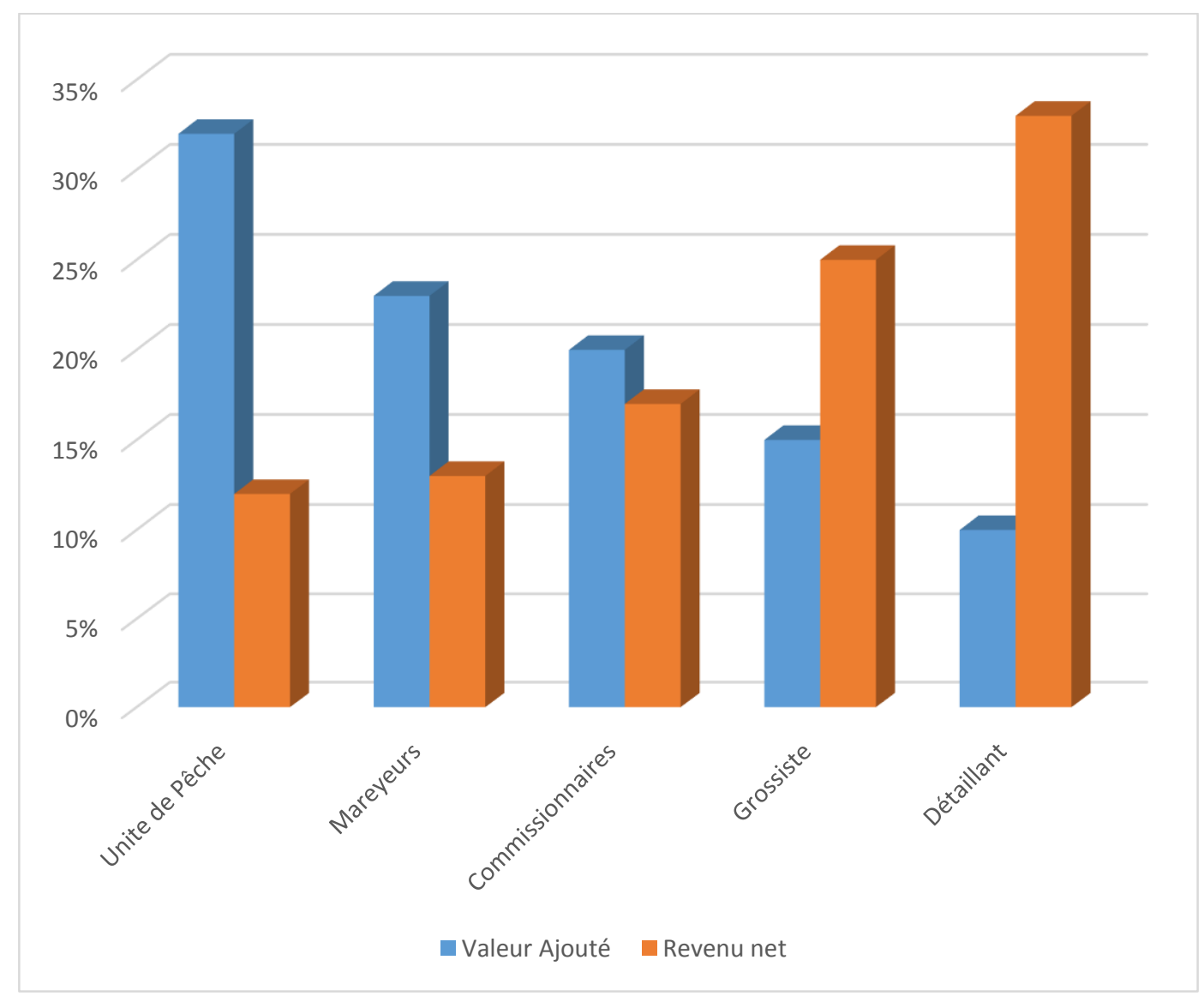

Figure 5 : Contribution des acteurs de la filière Bagrus dans la création de la valeur ajoutée totale. 


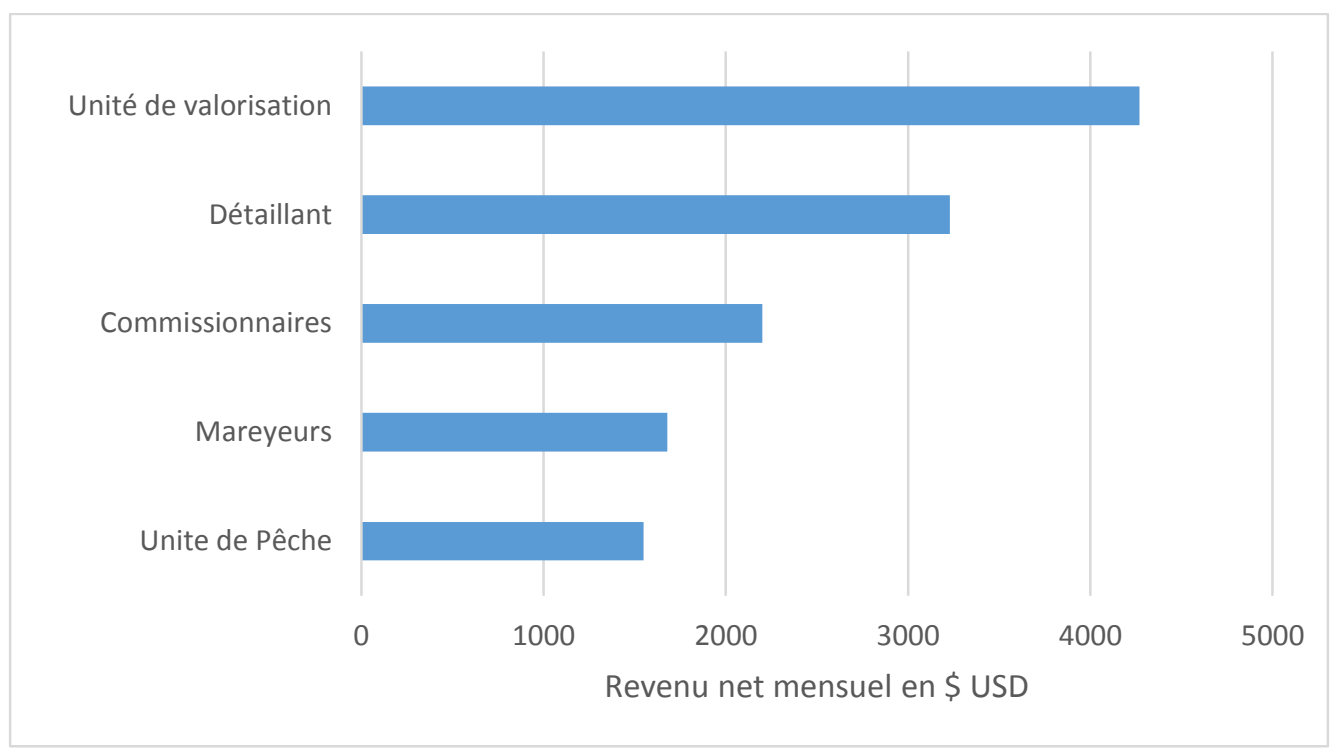

Figure 6 : Rentabilité économique des différents acteurs de la chaîne de valeur Bagrus spp aux Lacs Edouard et Albert (LAE).

Tableau 3 : Contraintes et opportunités identifiées chez les acteurs de la chaîne de valeur dans l'exploitation de Bagrus spp aux LAE.

\begin{tabular}{|c|c|c|c|}
\hline Maillons & Acteurs & Contraintes & Opportunités \\
\hline Production & Pêcheurs & $\begin{array}{l}\text { - Manque de crédit spécifique ; } \\
\text { - Accidents de travail et } \\
\text { maladies; } \\
\text { - Inexistence de contrat de } \\
\text { service écrit en vue de } \\
\text { sécuriser les droits des } \\
\text { contractants ; } \\
\text { - Manque de fond de roulement } \\
;\end{array}$ & $\begin{array}{l}\text { - Disponibilité de Bagrus } \\
\text { spp pour la consommation } \\
; \\
\text { - Existence de marché } \\
\text { d'écoulement avec une } \\
\text { demande locale forte ; } \\
\text { - Organisation des acteurs } \\
\text { - Création de coopérative } \\
\text { d'épargne; }\end{array}$ \\
\hline Intermédiaires & Commissionnaires & $\begin{array}{l}\text { - Manque de crédit spécifique ; } \\
\text { - Inexistence d'appui / } \\
\text { intervention ; } \\
\text { - Matériels / équipements peu } \\
\text { moderne ; } \\
\text { - Inexistence de logistique de } \\
\text { stockage à long terme ; }\end{array}$ & $\begin{array}{l}\text { - Disponibilité de poisson pour } \\
\text { la consommation ; } \\
\text { - Existence de marché } \\
\text { d'écoulement avec une } \\
\text { demande intérieure forte ; } \\
\text { - Existence de logistique de } \\
\text { stockage à long terme ; }\end{array}$ \\
\hline
\end{tabular}


- Forte concurrence du Bagrus - Formation sur les différents spp frais avec les Tilapia types de stockage et de importés ; conservation du poisson frais.

- Faible niveau d'organisation.

\section{Commercialisation}

\section{DISCUSSION}

Facteurs de production et mode d'exploitation de Bagrus spp. aux lacs Edouard et Albert (LEA)

Aux lacs Edouard et Albert se pratique la pêche artisanale avec la pirogue monoxyle comme principal type d'embarcation utilisé pour la capture de Bagrus spp. Le filet maillant y reste l'engin de pêche le plus utilisé pour la capture du Bagrus spp. suivi de palangres comme signalé par le SENADEP (2019) dans le rapport national de l'enquêtes cadre 2018 réalisé aux LEA. Certains pêcheurs rencontrés utilisent les barques et les palangres. Pour cette catégorie de pêcheurs, le fait de leur interdire l'accès aux zones de frayères est un facteur limitant pour leur activité étant donné que c'est au niveau des frayères qu'ils s'approvisionnent en appâts. Cette pratique illégale est à décourager et serait l'une des raisons qui sont à la base de la baisse de la production de poisson tout en compromettant l'avenir du lac.
La quasi-totalité des pêcheurs vendent du Bagrus à l'état frais. Cependant, pour des raisons de conservation, Bagrus est transformé à l'état salé ou fumé et c'est sous cet état que bon nombre de grossistes et détaillants le vendent. Pour la plupart des transporteurs, Bagrus est transporté à l'état salé au lac Albert et à l'état fumé au lac Edouard. Or, le fumage comme technique de conservation nécessite du bois de chauffage, une denrée pourtant rare tant sur le lac Albert que sur le lac Edouard. Son utilisation implique un déboisement des milieux environnant les lacs, tout ceci avec ses conséquences non négligeables sur le plan environnemental. Pire encore, le lac Edouard qui a une particularité par rapport au lac Albert. Le lac Edouard ainsi que ses pêcheries font partie intégrante du Parc National des Virunga (PNVi), un site inscrit à la liste des sites du patrimoine mondial et où toutes sortes de cultures sont formellement interdites. Les populations situées dans les enclaves cherchent 
ainsi du bois non comme source d'énergie pour leurs ménages mais aussi pour le fumage du poisson dans des forêts du parc en violation des objectifs de conservation des ressources naturelles. Se référant à cette interaction entre l'homme exploitant les ressources halieutiques et l'environnement, on pourrait se dire que la pêche constitue sous cet angle une menace pour l'environnement. Nos résultats sont également soutenus par Musubaho (2011).

\section{Aspect organisationnel de la commercialisation}

Les quantités de poisson achetées varient selon le type d'acteur et le mode de transport des captures. La conservation (aussi bien pendant la pêche et la collecte que pendant le transport et la vente dans les centres de consommation) se fait par fumage, salage et par froid. De peur d'engager des coûts supplémentaires, plus de $80 \%$ des pêcheurs préfèrent vendre leurs poissons à l'état frais et la transformation commence au niveau des détaillants et des grossistes (Figure 3). La majorité des pêcheurs traditionnels n'utilisent pas de glace ou ne respectent pas les normes de conservation du fait de manque de source d'alimentation au système de froid dans la région. D'après la FAO (2009), il faut $1 \mathrm{~kg}$ de glace pour conserver $2 \mathrm{~kg}$ de poisson. On observe la même chose chez les détaillants du petit marché. En effet, le détaillant expose son produit frais sans glace dans un plateau métallique installé sur les étalages. Il est évident que dans cette situation, les poissons frais doivent être écoulés rapidement, dans un délai ne dépassant pas un jour. Mais, heureusement, les espèces carnivores telles que Bagrus spp. s'altèrent moins vite que les espèces herbivores comme, Tilapia spp, Barbus spp., etc. (Nyebe et al., 2015 ; Okito et al.2017a). Cependant, le mode de vente en détail et le système à tour de rôle obligent respectivement les grossistes-intermédiaires et les grossistes-transporteurs à se munir des congélateurs pour conserver leurs produits.

Les pêcheurs, bien qu'étant les acteurs principaux de la pêche, sont lésés dans la répartition des revenus parce qu'ils n'ont pas accès aux marchés de consommation. Ce résultat est proche de ceux obtenus au Lac Tanganyika pour lequel le circuit de commercialisation est monopolisé par les mareyeuses qui imposent les règles du jeu (Mushagalusa et al., 2015 ; Okito et al., 2017). Les agents économiques de la commercialisation de Bagrus spp. utilisent plus les circuits courts et intégrés contrairement aux détaillants des points de vente et aux grossistes des marchés urbains, où tout le monde emprunte les circuits longs. Par ailleurs, même si le circuit long pourrait être le plus rentable, il n'est pas exclu qu'il pourrait souvent être le plus risqué car l'ensemble de la cargaison peut s'altérer à cause de la distance ou d'éventuelles pannes techniques. Ces résultats corroborent ceux de Dominique et al. (2013), qui affirment que la denrée étant hautement périssable, l'immobilisation de ces véhicules en cours de route par des pannes ou autres causes peut entrâner la détérioration et la perte de toute la cargaison.

\section{En rapport avec les rôles et relations des acteurs de la chaîne de valeur}

Dans ces deux lacs, les différents acteurs animant la chaine de valeur autour de la pêche de Bagrus spp. sont principalement les pêcheurs, les mareyeuses, les commissionnaires, les commerçants (grossistes et détaillants) et les consommateurs. La pêche est donc une activité économique qui a réussi à dominer une masse importante de la population active. La production immédiate des investissements y placés serait le facteur moteur de cette attraction massive, contrairement au secteur agricole dont la production exige une durée relativement longue pour en jouir (Musubaho, 2011).

Les résultats de cette étude ont montré une forte dépendance des pêcheurs à l'égard des mareyeurs et commissionnaires. En effet, pour assoir leur domination, les mareyeurs jouent un rôle important dans la couverture des besoins des pêcheurs en fonds de roulement. Il est fréquent de rencontrer un mareyeur 
accordant aux pêcheurs des avances pour l'achat du carburant, des vivres, des appâts, du matériel de pêche, et pour régler les dépenses d'entretien des moyens de production. Cette assistance continue devient cruciale en période de faible productivité de la pêche ou d'inactivité totale. Par conséquent, le pêcheur demeure redevable au mareyeur et ne peut se passer de ses services. Et pour cause, le pêcheur étant dans l'obligation de traiter avec le même mareyeur jusqu'à remboursement total de ses créances, se voit contraint de lui remettre sa production à des conditions qui sont davantage dictées par le mareyeur que par le jeu de la loi de l'offre et de la demande. On pourrait en déduire qu'il s'agit là de l'une des raisons qui expliquent la survie des transactions informelles même dans les situations où les facilités logistiques et commerciales ne font pas défaut. Cette typologie de la chaîne de valeur étudiée se rapproche de celle rencontrée dans la plupart des chaînes des valeurs de piscicultures et de pêches à petites et moyennes échelles (Amian et al., 2018 ; FAO, 2014). L'analyse de la chaîne de valeur montre que les relations existant entre les différents acteurs internes sont de nature informelle.

Les conclusions de notre étude confirment l'hypothèse selon laquelle les intermédiaires occupent un rôle clé dans la filière de pêche artisanale, au détriment du maillon primaire représenté par le pêcheur. Par rapport aux travaux antérieurs cités précédemment, nos résultats corroborent les conclusions des travaux de Davidsson (2007) qui ont abouti au constat que la majorité des bénéfices sont capturés par le commerce de gros, le commerce de détail et le secteur de la transformation. Dans le cas de la filière de pêche artisanale marocaine, $65 \%$ des revenus reviennent à des agents autres que les unités de pêche. Ces constatations vont dans le sens des conclusions de la FAO (2020); Gudmundsson et al., (2006) et Bjorndal et al., (2014) qui avancent que les petits pêcheurs reçoivent les bénéfices économiques les plus faibles par rapport aux autres agents de la chaîne des valeurs.

Une dynamique est en passe de prendre de l'ampleur : face aux difficultés croissantes, résultant notamment du recul du niveau d'abondance des ressources, les pêcheurs artisans, en proie à des difficultés financières, sont, de plus en plus, la cible des convoitises des mareyeurs.

\section{Contraintes et opportunités identifiées chez les acteurs de la chaine de valeur}

La faible compétitivité économique des pêcheurs dans le fonctionnement de la chaîne des valeurs serait aussi liée à l'inexistence de liens de service formalisés avec les autres acteurs de la chaîne mettant en relief les difficultés énoncées dans cette étude. Ces aspects contribuent davantage à la vulnérabilité des différents acteurs primaires de la chaîne et particulièrement les pêcheurs. Or, il est montré que les chaînes de valeur agricoles fonctionnent de manière plus efficace lorsque les exploitants agricoles ont établi des partenariats avec les fournisseurs d'intrants et de services et avec les entités qui commercialisent et transforment leur production (Westlake, 2014). De cette perspective, le renforcement des liens et relations entre les acteurs de la chaîne de manière inclusive permettraient d'atténuer les contraintes, d'améliorer les services et d'accroître la compétitivité économique des producteurs. Aussi, le développement des opportunités identifiées et l'amélioration de la gouvernance tout le long de la chaîne permettraient d'augmenter la performance de la chaîne de valeur de la filière d'exploitation de Bagrus spp. aux lacs Albert et Edouard en République Démocratique du Congo.

Dans ces régions, la pêche artisanale est une activité de diversification des économies de plantations familiales et contribue à la chaine de valeur locale (Matunguru,2013; Cikwanine, 2014). La promotion des systèmes locaux d'innovation piscicoles dans une approche d'intensification écologique constituerait un gage de développement 
durable de ces économies (Oswald et al., 2015). Au niveau des liens avec les autres entités, l'appui des acteurs de la recherche et du développement aux producteurs pourrait être réalisé plus efficacement dans un cadre global à travers l'approche Recherche Agricole Intégrée pour le Développement (RAID). En effet, cette approche basée sur la mise en place de plateforme d'innovation réunissant l'ensemble des intervenants (producteurs, chercheurs, conseillers agricoles, transformateurs, fournisseurs d'intrants, commerçants) de la chaîne de valeur permet d'optimiser les performances de chaque acteur (CORAF/WECARD, 2014). Les chaînes de valeur n'existent pas séparément ; elles sont intégrées dans un environnement social, économique, culturel et politique hautement complexe, qui détermine la nature et le succès des opérations commerciales au sein de la chaîne de valeur (Herr et Muzira, 2009). De ce point de vue, les politiques de développement devraient intégrer la pêche en général et l'exploitation de Bagrus spp. en particulier dans l'élaboration des programmes de développement de la pêche artisanale en prenant en compte les spécificités des systèmes de production, l'environnement socioéconomiques des acteurs, le renforcement des compétences locales et des savoir-faire existant.

\section{Conclusion}

Cette étude sur la chaîne de valeur de la filière de pêche de Bagrus spp. Aux lacs Albert et Edouard a été une première tentative approfondie visant à analyser les mécanismes de création et de distribution de la Valeur Ajoutée à la lumière des modes d'organisation et de gouvernance qui régissent les rapports entre les agents de cette filière. Il en ressort que trois types de chaînes de valeurs ont été identifiées : les chaînes de valeurs tournées vers le marché local, les chaînes de valeurs urbaines et les chaînes de valeurs à caractère mixte dont la production sera destinée tantôt au marché à l'export, tantôt au marché domestique. Le modèle de gouvernance qui domine dans ces chaînes s'apparente à un "réseau dirigé" avec de nombreuses situations où la gouvernance de la chaine de valeur possède les caractéristiques d'un "réseau équilibré". Le pêcheur est le principal générateur de valeur ajoutée. Toutefois, la dynamique de la filière est orchestrée par les intermédiaires qui développent des stratégies où se mêlent confiance, compétition et domination. Etant à la fois clients, concurrents, associés et créanciers des pêcheurs, leur rémunération est à la hauteur de leur statut étant donné qu'ils captent la plus grande part des revenus distribués dans la filière. La durabilité de la pêche artisanale exige une répartition plus équilibrée et plus équitable des bénéfices tirés de l'exploitation des ressources halieutiques. Maillon faible de la chaîne de valeurs, le pêcheur artisanal devrait voir son statut rehaussé face à ses partenaires. A côté des investissements physiques qui visent à améliorer l'environnement de la pratique de pêche et des transactions commerciales, l'intégration constitue une voie d'amélioration à encourager. Enfin, comme l'étude s'est intéressée aux chaînes de valeurs dans la limite d'un seul genre de poisson, les maillons qui se déroulent en dehors de l'exploitation de Bagrus spp. n'ont pas été abordés. Or, puisqu'il s'agit souvent de produits frais et que ce sont les derniers maillons de la filière qui procurent le plus de valeur, la contribution du segment de la vente au détail est donc sous-estimée pour les chaînes de valeurs destinées aux marchés urbains. Des études portant sur la totalité de la chaîne globale de valeur de l'ensemble de la pêche artisanale permettront d'améliorer les résultats de ce travail.

\section{CONFLIT D'INTERETS}

Les auteurs déclarent qu'il n'y a aucun conflit d'intérêts.

\section{CONTRIBUTIONS DES AUTEURS}

JMM est intervenu dans toutes les phases de l'étude. Il s'agit de la conception de l'étude, la collecte des données, le dépouillement, le traitement et l'analyse des 
données et la rédaction du présent manuscrit ; GMO est intervenu dans la conception de l'étude, le dépouillement, le traitement, l'analyse des données et la rédaction du présent manuscrit. PKK, JJA et GTH sont intervenus dans la collecte des données, le dépouillement, le traitement et l'analyse des données, JCM, GN et VNM sont intervenus dans la conception de l'étude et dans la correction du manuscrit.

\section{REMERCIEMENTS}

Nous remercions le personnel du Projet LEAF II pour son support administratif. Nos remerciements vont aussi, aux enquêteurs de pêche ainsi qu'aux pêcheurs des Lacs Edouard et Albert pour leur franche collaboration.

\section{REFERENCES}

Amian AF, Melecony CB, Wandan EN, Vanga AF, Imorou TI, Kaudhjis PJA, Ewoukem ET. 2018. Analyse de la chaine de valeur de la pisciculture extensive familiale en Côte d'Ivoire : cas des régions du CentreOuest et Sud-ouest. Journal of Applied Biosciences, 131: 13335 - 13346.

Balole BLE, Mumbere JC, Matunguru MJ, Kujirakwinja D, Shamavu E, Muhindo P, Tchouamo IR, Baudouin M, Micha JC. 2018. Production et impacts de la pêche dans la partie congolaise du Lac Edouard. Tropicultura, 36(3) : 539-552.

Bockel L, Tallec F. 2005a. Commodity chain analysis. Constructing the commodity chain functional analysis and flow charts. Rome: FAO. EASYPol, module 43. http://www.fao.org/docs/up/easypol/330/ value_chain_analysis_flow_charts_043e n.pdf

Bockel L, Tallec F. 2005b. Commodity chain analysis. Financial analysis. Rome: FAO. EASYPol,module 44 .

http://www.fao.org/docs/up/easypol/331/ value_chain_analysis_financial_analysis 044en.pdf

Cikwanine KD. 2014. La pêche au lac Albert: Statistiques des engins et analyse de l'impact des activités de pêche sur la biodiversité du lac Albert. Rev. Shalom. Dév., 2(1) : 8-36.

CORAF/WECARD. 2014. Mise en place et Animation des plateformes d'innovation suivant l'approche IAR4D. 56p.

Dominique B, Olivier R, Bernardoni P, Bagal M, Giovanni B, Mrescotti A, Giulia O, Laniau M, Bougouin H. 2013. Etude du potentiel de la commercialisation des produits agricoles des pays ACP (Afrique, Caraïbes, Pacifique) utilisant les indications géographiques et les marques avec origine (ACP-AGGI). IRAM, Union Européenne, $153 \mathrm{p}$.

FAO. 2009. Vue générale du secteur national des pêches en République Démocratique du Congo. Profils de pêche et de l'Aquaculture par pays. FID/CP/RDC, FAO 2009, Rome, Italie, 70 p.

FAO. 2020. La situation mondiale des pêches et de l'aquaculture. FAO, Rome, Italie, $247 \mathrm{p}$.

Fon DE, Nyebe IM, Meutchaeye F, Jaza AF. 2015. Cost Analysis of fish smoking in Cameroon. Journal of Animal Poduction Advances, 5(11): 794-800. DOI: http://dx.doi.org/10.5455/japa.20151111 075937

Gereffi G, Korzeniewicz M. 1994. Commodity chains and global capitalism. Westport, CT: Praeger ; 1-14.

Herr M, Muzira T. 2009. Le développement de la chaîne de valeur chaînes de valeur au service du travail décent : Guide à l'usage des praticiens du développement, des gouvernements et des entreprises privées. Genève: Bureau international du Travail ; 239 p.

Kaplinsky R, Morris M. 2001. A handbook for value chain research. IDRC: Brighton.

Naji M, Araba A, Majid B, Dehhaoui M. 2015. Gouvernance et revenus dans la filière de pêche artisanale marocaine : analyse par la chaîne de valeur. New Medit, 4(2015): 11-18.

Luhusu KF, Micha JC. 2013. Analyse des modes d'exploitation des ressources halieutiques du lac Maï-Ndombe en 
République Démocratique du Congo. Geo-Eco-Trop., 37(2): 273-284.

Manala AC, Okito MG, Lubunga DP, Kwibe A. 2020. Commercialization of Fish and their consumption rate by the population of Uvira, South Kivu, DR Congo. International Journal of Innovative Science and Research Technology, 5(1): 1147- 1157.

Matunguru J. 2013. Rapport du dénombrement exhaustif des populations humaines riveraines du lac Edouard. ACFUK/ICCN-PNVi, UNESCO, 58p.

Mulongaibalu M, Muderhwa N, Mujugu E, Chapman L, Efitre J, Bwanika G. 2015. Identification of migratory and spawning habitats of Clarias gariepinus (Burchell, 1 822) in Lake Edward - Ishasha River watershed, Albertine Rift Valley, East Africa. Int. J. Fish. Aquat. Stud., 2(3): 128-138.

Mushagalusa., Micha JC, Ntakimazi G, Muderhwa N. 2015. Brief evaluation of the current state of fish stocks landed by artisanal fishing units from the extreme northwest part of Lake Tanganyika. Int. $J$. Fish. Aquat. Stud., 2(4) : 41-48.

Musubaho K. 2011. Gestion des ressources halieutiques et problématique du développement sur les côtes Nord et Ouest du lac Edouard en République Démocratique du Congo. Mémoire présenté et défendu en vue de l'obtention du Diplôme d'Etudes Approfondies (DEA) en Economie Rurale, U.C.G., $133 \mathrm{p}$.

Nahayo A. 2010. Analyse de la Filière pêche. Projet de Renforcement de la filière du conditionnement et de transformation du poisson du Lac Tanganyika et appui à sa commercialisation, FAO, 76 p.
NELSAP. 2019. Rapport Technique Régional des Enquêtes d'Evaluation des Captures (EEC) menées sur les côtes de la République de l'Ouganda et de la République Démocratique du Congo (RDC) des lacs Edouard et Albert entre Juillet et Août 2019 par NaFIRRI et SENADEP, $137 \mathrm{p}$.

Okito MG, Micha JC, Habarugira JB, Ntakimazi G, Nshombo MV, Bizuru NP, Muhirwa BG. 2017. Socio-économie de la pêche artisanale dans les eaux burundaises du lac Tanganyika à Mvugo et Muguruka. International Journal of Biological and Chemical Sciences, 11(1): 247-265.

DOI: http://dx.doi.org/10.4314/ijbcs.v11i1.20.

Oswald M, Mikolasek O, Meké P, Blé MC, Ewoukem ET, Vanga FA, Imorou TI, Eyango TM. 2015. Lessons learnt from a review of extensive fish farming inside family plantations economies through West Africa and their contribution to the local value chain. 5th International Symposium for Farming System Design. 7-10 September 2015, Montpellier, France. 525-526.

Petit P. 2006. Les pêches dans la partie congolaise du lac Edouard : Analyse de la situation actuelle. Vredeseilanden (VECO) Blijde Inkomstraat 50, 3000 Leuven, Belgium, $54 \mathrm{p}$.

Vakily J. 1989. Les pêches dans la partie zaïroise du Lac Idi Amin. Analyse de la situation actuelle et potentiel de développement. Rapport Technique de Pêche au Zaïre. Gouvernement de la République du Zaïre. Département des Affaires Foncières, Environnement et Conservation de la Nature. Commission des Communautés Européennes, 45 p. 